Discussion/conclusion Education is key to increase awareness about the importance of HIV screening. Healthcare professionals should be aware of and address barriers to screening during consultations. An educational poster has been produced for the clinic with the intention of increasing awareness of HIV among the pregnant population.

\section{P221 A SURVEY EXAMINING HEALTH SEEKING BEHAVIOURS OF THOSE ACCESSING SEXUAL HEALTH SERVICES IN LONDON}

Marie McNulty*, Jake Bayley, Michael O'Hanlon, Jennifer Hong, Rebecca O'Connell. Barts Health NHS Trust, London, UK

\subsection{6/sextrans-2016-052718.269}

Background/introduction Open access to sexual health services reduces STI's and onwards transmission. Given cuts to Public Health budgets, a better understanding of how patients access care is vital to rationalise services.

Aim(s)/objectives Assessing demographics and health behaviours of sexual health clinic attendees to improve service delivery.

Methods Patient-directed questionnaires were completed on registration in a London Trust. Information including demographics, travel times and whether patients sought help before attending were collected.

Results 231 surveys were returned with respondents 48\% white, $23 \%$ black and $13 \%$ Asian. $62 \%$ of patients walked-in, $34 \%$ booked online. 52/217 (24\%) sought advice from elsewhere before attending (primarily GP - 26/52, 50\%) with 50\% finding it useful. Of 107 responses, 41 (38\%) tried self-treating before attendance. Symptomatic patients were more likely then asymptomatic patients to seek help elsewhere (40/113 versus 12/99, $\mathrm{p}<0.05)$. No significant differences in behaviour were observed given age, ethnicity or employment, or previous STI $<12$ months ago. No patients with qualifications less than GCSE sought prior to attendance. $80 \%$ of patients travelled under 30 minutes to clinic, $58 \%$ attended their closest clinic.

Discussion/conclusion Our data demonstrates the clinics surveyed serving a very local population. However a significant proportion of patients, particularly those with symptoms, seek help elsewhere before attending, with only 50\% finding this useful. This highlights the importance of specialist services addressing local patients' health needs. Overall socio-demographic factors did not appear to influence health seeking behaviour, although those with a lower education status appeared to access services more directly.

\section{P222 DOES HIV INFECTION INCREASE COMPLICATIONS AFTER INTRA UTERINE CONTRACEPTION (IUC)?}

Savidya Adikari* ${ }^{*}$ Mannampallil Samuel. King's College Hospital, London, UK

\subsection{6/sextrans-2016-052718.270}

Background As estimated by World Health Organisation about $50 \%$ of all HIV infected individuals are women. Comparison data for complications after IUC in HIV positive and negative women are lacking.

Aims The aim of our study is to compare short-term complications and side-effects after IUC in the above two groups.

Methods Retrospective notes review of 76 patient records of HIV negative women who attended in 2013, for IUC and followed up for 3 months was carried out. All HIV positive women, who attended between 2012-2015, for IUC and followed up within 3 months were included. Data including demographics and complications were collected in addition to HIV related parameters.

Results Among the 49 HIV positive women 46 were on treatment and was undetectable at the time of IUC insertion. Mean CD4 count was 589 cells $/ \mu \mathrm{L}$. Mean age was 38 years, and 30 in the negative women. Black ethnicity was common among both groups. Pelvic pain was reported in $6 \%$ of the HIV group vs $17 \%$ of the non-HIV group (P value $=0.034$ ). Incidence of lost threads was also significantly high in HIV negative women (P value $=0.018) .31 \%$ of the HIV group reported heavy or prolonged bleeding vs $37 \%$ of the negative group.

Discussion In HIV negative women, pelvic pain and incidence of lost threads were significantly high. Occurrence of any complication or side effect was also significantly high in HIV negative women $(\mathrm{P}$ value $=0.022)$.

\section{P223 PROVISION OF MENTAL HELATH CARE IN HIV POSITIVE HOSPITAL INPATIENTS EVALUATED AGAINST BRITISH PSYCHOLOGICAL SOCIETY (BPS) STANDARDS}

${ }^{1}$ Tatyana Sahabandu*, ${ }^{1}$ Rosanna Grimes, ${ }^{1,2}$ Alice Bennett, ${ }^{1}$ Philip Henshaw, ${ }^{1}$ Daniel Bradshaw. 'Brighton and Sussex University Hospitals NHS Trust, Brighton, UK; ${ }^{2}$ Sussex Partnership NHS Foundation Trust, Brighton, UK

\subsection{6/sextrans-2016-052718.271}

Background The prevalence of mental health problems (MHP) in PLWH is significantly higher than in the general population. Little is known of the prevalence of MHP and experience of patients of $\mathrm{MH}$ services locally.

Aim This study aimed to audit the provision of $\mathrm{MH}$ care in HIV-positive inpatients locally against 2011 BPS standards.

Methods We undertook a retrospective notes review of HIV-positive inpatients between 15/07/2015 - 30/11/2015. The following data were collected: demographics, HIV parameters, substance misuse, and $\mathrm{MH}$ history. Phone interviews were held to obtain feedback on patients' experiences of MH care. Statistical analysis was undertaken using chi-square or Fisher's exact test.

Results Of seventy-three patients $86 \%$ were male $(n=63)$ and $80 \%$ UK-born Caucasian ( $\mathrm{n}=58)$. Median CD4 was 495 cells/ mm3 (range: 8-1847); HIV-1 viral load was undetectable in $78 \%(\mathrm{n}=57) ; 8 \%$ were HCV antibody positive $(\mathrm{n}=6) ; 3 \%$ homeless $(n=2), 26 \%$ reported alcohol excess $(n=19), 4 \%$ injection drug use (IDU) ( $\mathrm{n}=3), 32 \%(\mathrm{n}=23)$ active and $38 \%$ $(\mathrm{n}=28)$ previous MHP. Only IDU was significantly associated with active MHP, $(\mathrm{p}=0.01)$. Of 15 patients reporting MHP who provided feedback, 6 (40\%) felt healthcare professionals (HCPs) had not given them sufficient opportunity to discuss their psychological wellbeing and $40 \%(n=6)$ reported experiencing stigma from HCPs.

Conclusion Results suggest a high prevalence of MHP in this cohort. Many reported difficulties discussing MHPs with HCPs. We have therefore initiated a pilot joint HIV/Psychiatric clinic to improve access to $\mathrm{MH}$ services.

\section{P224 MISSED OPPORTUNITIES AND COST IMPLICATIONS IN A HIV LOW PREVALENCE REGION IN THE UK}

Darshanie Mallikarachchi*, Amy Beswetherick, Jane Holder, Sathish Thomas William. Taunton and Somerset NHS Foundation Trust, Taunton, UK 
Background/introduction Missed opportunities is a leading cause for late presentation in HIV.

Aim(s)/objectives We analysed missed opportunities, clinical outcomes and associated cost in a HIV low prevalence region in UK.

Methods A retrospective review of case notes and pathology system of new HIV diagnosis from 2010 to 2013 was undertaken. Clinical summary preceding 12 months of diagnosis collected from GPs with patient's consent. Data analysed using Excel workbook.

Results Out of 25 new HIV diagnosis, 17 males, 21 white ethnicity, 10 heterosexual and 6 bisexual. One third $>40$ years. Sixteen $(64 \%)$ were late diagnosis with CD4 < 350. 13/16 had CD4 < 200 and 9/16 (56\%) had an AIDS defining illness. 19/25 (76\%) had atleast one missed opportunity (range 1-16). 11/19 in primary care and remaining at different levels. There was no difference in VL between early and late diagnosis. 10/16 had a blood test in the preceding 12 months. In the first 12 months post diagnosis, early group had 51 clinical consultation compared to 147 in late group. Three patients had extended inpatient stay in the late group.One died. Using Reference costs of around $£ 385$, late diagnosis costed $£ 56595$ compared to $£ 19635$ for early excluding inpatients cost, excess bed days, additional outpatient investigations, medications including ARVS and other specialty referral costs.

Discussion/conclusion Our study shows increased missed opportunities in apparently non high-risk groups resulting in poor outcomes and significant costs. Higher HIV awareness and national testing policy tailored to HIV low prevalence region is required.

\section{P225 LYMPHOGRANULOMA VENEREUM: CASE REPORT OF SIMULTANEOUS PENILE ULCERATION AND BUBO FORMATION}

Elizabeth Okecha*, Ashish Sukthankar. Central Manchester University Hospitals NHS Foundation Trust, Manchester, UK

\subsection{6/sextrans-2016-052718.273}

Background/introduction Lymphogranuloma venereum (LGV) has become an important UK public health issue since 2003. HIV positive men who have sex with men (MSM) continue to be the predominant risk group for contracting LGV in the UK.

Aim(s)/objectives LGV commonly presents as proctitis alone; we present an unusual case of a patient who presented with simultaneous rectal symptoms, penile ulceration and bubo.

Methods A 25 year old HIV (CD4 996 (43\%)/viral load undetectable) and Hepatitis C co-infected MSM presented to A\&E with a 2 week history of painful left inguinal swelling. Additional history revealed episodes of bloody diarrhoea which the patient related to a previous diagnosis of Inflammatory Bowel disease. Examination revealed a left inguinal bubo and 3 areas of penile ulceration (2 painful, one indurated/painless). Proctoscopy was normal. Empirical treatment with Doxycycline and Aciclovir was commenced.

Results Nucleic acid amplification testing (NAAT) was performed on pharynx, rectum, urine and penile ulcers. Urine NAAT was positive for Chlamydia trachomatis; penile ulcer NAAT was positive for LGV specific DNA. Herpes simplex and Treponemal PCR from the ulcers were negative. Rectal NAAT was negative for Chlamydia trachomatis. An Ultrasound of the left inguinal bubo revealed abscess formation with frank pus seen on aspiration.
Discussion/conclusion LGV is commonly associated with proctitis and genital ulcers of LGV are evanescent. Persistence of LGV ulceration until bubo formation has not been reported to-date. The negative Rectal NAAT for Chlamydia was unusual however the diarrhoeal symptoms settled with Doxycycline.

\section{P226 REATTENDANCE, RETESTING AND TEST POSITIVITY AMONG PATIENTS INITIALLY TREATED FOR CHLAMYDIA OR GONORRHOEA IN A LARGE INNER CITY CLINIC}

Sarah Bradley*, John Saunders, Eva Jungmann. Mortimer Market Centre, CNWL, London, UK

\subsection{6/sextrans-2016-052718.274}

Background/introduction Reinfection with a bacterial sexually transmitted infection following treatment is common. National guidelines recommend retesting for chlamydia three to six months after treatment in $<25$ year olds.

Aim(s)/objectives To review reattendance, retest and positivity rates in patients with chlamydia or gonorrhoea at a large innercity clinic.

Methods Patients diagnosed with chlamydia or gonorrhoea were identified in our electronic patient records (March-September 2015). Proportion reattending, retesting and test positivity two to four months after treatment of the initial infection were recorded.

Results The minority of patients treated for chlamydia and gonorrhoea reattended and retested for infection (Table 1). Positivity was high in those who retested, especially in MSM. Two HIV diagnoses and 89 syphilis serology positive results were found in those retesting.

Abstract P226 Table 1 Reattendance, retesting and test positivity among patients initially treated for chlamydia or gonorrhoea, by age and risk group

\begin{tabular}{lllll}
\hline & $<25$ years $(n=584)$ & $\geq 25$ years $(n=1,713)$ \\
\hline & $\begin{array}{l}\text { Heterosexual } \\
(n=442)\end{array}$ & $\begin{array}{l}\text { MSM } \\
(n=142)\end{array}$ & $\begin{array}{l}\text { Heterosexual } \\
(n=647)\end{array}$ & $\begin{array}{l}\text { MSM } \\
(n=1066)\end{array}$ \\
\hline $\begin{array}{l}\text { Total: Chlamydia } \pm \\
\text { Gonorrhoea }(\mathrm{n}=2297)\end{array}$ & & & & \\
Reattended & $97(22 \%)$ & $42(30 \%)$ & $148(23 \%)$ & $481(45 \%)$ \\
$\begin{array}{l}\text { Retested } \\
\text { Positivity }\end{array}$ & $76(78 \%)$ & $25(60 \%)$ & $96(65 \%)$ & $258(54 \%)$ \\
\hline & $11(14 \%)$ & $9(36 \%)$ & $11(11 \%)$ & $92(36 \%)$ \\
\hline
\end{tabular}

Discussion/conclusion Retesting rates following treatment for chlamydia and gonorrhoea are low. Those who did reattend and retest experienced high rates of infections and represent an important group to target for active recall.

\section{P227 PATIENT SATISFACTION - NEW LGBTQ (LESBIAN, GAY, BISEXUAL AND QUEER) SEXUAL HEALTH CLINIC}

Grainne Cooney*, Eleanor Hamlyn, Sabrina Wallace, Ella Svensson, Michael Underwood, Tristan Barber. John Hunter Clinic, Chelsea and Westminster Hospital., London, UK

\subsection{6/sextrans-2016-052718.275}

Background/introduction This clinic opened in 2015 offering a weekly specialist sexual health service for our LGBTQ community. Burden of pathology is high, suggesting a potentially more 